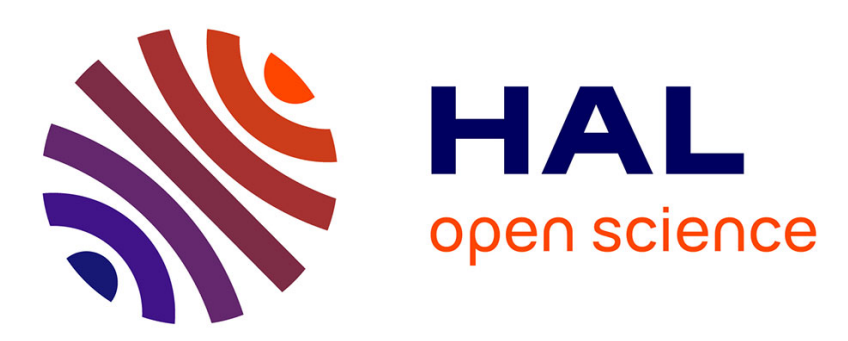

\title{
Analyse harmonique adaptée aux signaux à modulation de fréquence hyperbolique
}

\author{
Stéphane Puechmorel, B. Lacaze
}

\section{To cite this version:}

Stéphane Puechmorel, B. Lacaze. Analyse harmonique adaptée aux signaux à modulation de fréquence hyperbolique. Journal de Physique IV Proceedings, 1994, 04 (C5), pp.C5-1371-C5-1374. 10.1051/jp4:19945305 . jpa-00253020

\section{HAL Id: jpa-00253020 https://hal.science/jpa-00253020}

Submitted on 1 Jan 1994

HAL is a multi-disciplinary open access archive for the deposit and dissemination of scientific research documents, whether they are published or not. The documents may come from teaching and research institutions in France or abroad, or from public or private research centers.
L'archive ouverte pluridisciplinaire HAL, est destinée au dépôt et à la diffusion de documents scientifiques de niveau recherche, publiés ou non, émanant des établissements d'enseignement et de recherche français ou étrangers, des laboratoires publics ou privés. 


\title{
Analyse harmonique adaptée aux signaux à modulation de fréquence hyperbolique
}

\author{
S. PUECHMOREL et B. LACAZE
}

ENSEEIHT-GAPSE, 2 Rue Camichel, 31071 Toulouse cedex, France

\begin{abstract}
The signals with an instantaneous frequency following an hyperbolic law are common in many fields of the signal processing, and particularly in the sturly of biologicals SONARS. In this paper we describe an harmonic analysis adapted to this kind of signals. The problem of stationnarity of stochastic processes will be adressed within the frame of the previous harmonic analysis. This allow us to define a power spectral density for second-order stationnary processes using the Bochner theorem. Some simulation results are presented showing the interest of the adapted harmonic analysis.
\end{abstract}

\section{Cadre de travail}

\subsection{Position du problème et analyse adaptée :}

Les signaux sur lesquels nous travaillerons ont une fréquence instantanée $f(t)=\frac{\omega_{0}}{2 \pi t}$. On peut donc écrire ces signaux sous la forme: $A e^{i \omega_{0} \log (t)}+b(t)$ où $b(t)$ est un bruit additif que l'on peut supposer blanc gaussien. Les paramètres importants du signal sont alors l'amplitude $A$ et $\omega_{0}$ la fréquence angulaire. Il est naturel dans ce cadre d'introduire un opérateur de filtrage adapté s'écrivant comme :

$$
T_{f}(\omega)=\int_{R^{+*}} f(t) e^{-i \omega \log (t)} d t(1)
$$

On vérifie bien sûr que dans ce cas, en prenant $f=A e^{-i \omega_{0} \log (t)} I_{K}$, où $I_{K}$ est l'indicatrice d'un intervalle borné de $R^{+*}$, le module carre de la transformée, $\left|T_{f}(\omega)\right|^{2}$, admet un maxinum global en $\omega=\omega_{0}$. Si l'on suppose maintenant que la fonction $f(t)$ est une sommme $f(t)=A e^{i \omega_{0} \log (t)}+b(l)$ ò̀ $b(t)$ est un processus de moyenne nulle, le résultat précédent reste vrai si l'on considère $\left|E\left[T_{f}(\omega)\right]\right|^{2}$.

Lopérateur (1) ne permet toutefois pas de construire une analyse harmonique. En effet, pour pouvoir considérer la transformation $F(f)$ :

$$
\hat{f} \rightarrow \hat{f}(\omega)=T_{f}(\omega)
$$

comme une transformée de Fourier, il est nécessaire que la fonction $e^{i \omega l o g(t)}$ intervenant dans l'expression de $T_{f}(\omega)$ présente les mêmes propriétés d'invariance que la mesure intégrale $d t$. 
Dans la suite nous allons montrer que le cadre naturel d'étude des signaux à modulation de fréquence hyperbolique est le groupe multiplicatif $R^{+*}$.

\subsection{Transformée de Fourier sur $R^{+*}$ :}

\subsubsection{Fonctions intégrables sur $R^{+*}$}

Une fonction $f(t): R^{+*} \rightarrow C$ est intégrable si :

$$
\int_{R^{+*}}|f(t)| \frac{d t}{t}<+\infty
$$

Cette définition de l'intégrale introduit une invariance par translation multiplicative. Il est à noter que les fonctions constantes ne sont pas intégrables, et que les fonctions de $L^{ \pm}\left(R^{+*}, \frac{d t}{t}\right)$ sont nécessairement nulles en 0 .

On définira également les fonctions appartenant à $L^{p}\left(R^{+*}, \frac{d t}{t}\right)$ par :

$$
f \in L^{p}\left(R^{+*}, \frac{d t}{t}\right) \Rightarrow \int_{R^{+*}}|f(t)|^{p} \frac{d t}{t}<+\infty
$$

On remarquera que $L^{2}\left(R^{+*}, \frac{d t}{t}\right)$ est un espace de Hilbert lorsque on le munit du produit scalaire :

$$
\langle f: g\rangle=\int_{R^{+*}} f(t) \overline{g(t)} \frac{d t}{t}
$$

Cet espace est ici l'équivalent pour $R^{+*}$ de l'espace des signaux à énergie finie.

\subsubsection{Convolution sur $R^{+*}$}

On appellera produit de convolution de $f$ et $g \in L^{1}\left(R^{+*}, \frac{d t}{t}\right)$ la quantité :

$$
f * g(\tau)=\int_{R^{+*}} f(t) g\left(\frac{\tau}{t}\right) \frac{d t}{t}
$$

si cette quantité existe. Le produit de convolution ainsi défini possède les mêmes propriétés de régularisation que le produit de convolution ordinaire, et peut être vu comme une opération de filtrage ( nous montrerons plus loin que l'on peut définir une transformation de Fourier dans laquelle ce filtrage possède une interprétation simple).

\subsection{3 fonctions de type positif}

Une fonction $f(t)$ définie sur $R^{+*}$ est dite de type positif si quelles que soient les constantes $c_{i} \in C$ et quels que soient $t_{i} \in R^{+*}$ on $\mathrm{a}$ :

$$
\sum_{i, j=1}^{n} c_{i} \overline{c_{j}} f\left(\frac{t_{i}}{t_{j}}\right) \geq 0
$$

Les fonctions des type positif admettent une densité spectrale de puissance ( théorème de Bochner généralisé ).

\subsubsection{Transformation de Fourier}

On appellera caractères du groupe multiplicatif $R^{+*}$ et l'on notera $\chi_{\omega}$ les solutions continues des équations :

$$
\begin{gathered}
\left|\chi_{\omega}(x)\right|=1 \\
\chi_{\omega}(x) \chi_{\omega}(y)=\chi_{\omega}(x y)
\end{gathered}
$$


on vérifie sans difficultés que l'on peut écrire : $\chi_{\omega}(x)=e^{i \omega \log (x)}, \omega \in R$.

La transformation de Fourier d'une fonction de $L^{1}\left(R^{+*}, \frac{d t}{t}\right)$ se définit alors par :

$$
\hat{f}(\omega)=\int_{R^{+*}} f(t) e^{i \omega l o g(t)} \frac{d t}{t}
$$

Cette transformation est linéaire et inversible.

\subsubsection{Propriétés de la transformée de Fourier et théoréme de Bochner}

- La transformation de Fourier transforme le produit de convolution précédemment défini en produit ordinaire. Cette propriété permet comme dans le cas de la transformation de Fourier usuelle de donner une interprétation fréquentielle du produit de convolution. Dans notre cas, le paramètre $\omega$ jone le rôle de la fréquence. Il est à noter que les méthodes classiques de calcul de filtre peuvent être transposées au cas présent.

- On peut définir comme dans le cas classique la transformation de Fourier des fonctions de $L^{2}\left(R^{+*}, \frac{d t}{t}\right)$. La transformation de Fourier est alors une isometrie de $L^{2}\left(R^{+*}, \frac{d t}{t}\right)$ dans $L^{2}\left(R^{+*}, \frac{d t}{t}\right)$. On peut écrire un équivalent de la formule de Parseval :

$$
\int_{R^{+*}} f(t) \overline{g(t)} \frac{d t}{t}=\int_{R} \hat{f}(\omega) \overline{\hat{g}(\omega)} d \omega
$$

\subsubsection{Le théorème de Bochner :}

La fonction d'autocorrélation d'un processus $X(t)$ défini sur $R^{+*}$ est :

$$
K_{X}(t, \tau)=E[X(t) X(t \tau)]
$$

Comme dans le cas des processus définis sur $R$, on appellera processus stationnaire au sens large un processus $X(t)$ tel que $K_{X}(t . \tau)=K_{X}(\tau)$. La fonction d'autocorrélation d'un tel processus est une fonction de type positif ( $\operatorname{sur} R^{+*}$ ) : elle admet une densité spectrale de puissance, $d \mu(\omega)$, telle que $K_{X}(\tau)=\int_{R} e^{i \omega l o g(\tau)} d \mu(\omega)$. L'analyse harmonique des processus stationnaires aul second ordre est donc parfaitement définie sur $R^{+*}$.

Il est à noter que les relations de Wiener-Lee et la formule des interférences existent sur $R^{+*}$, mais elles s'appliquent à des systèmes invariants par translation multiplicative.

\subsection{7 transformée de Fourier à fenêtre et à fenêtre glissante}

En pratique, la transformation précédente pose des problèmes de calcul au voisinage de 0 . Il est donc indispensable d'introduire des fenêtres de pondération limitant le support utile du signal à un intervalle $\left[\epsilon, x_{0}\right]$ avec $\epsilon>0$. La transformation devient alors :

$$
T(f)(\omega)=\int_{R^{+*}} f(t) w(t) e^{i \omega l o g(t)} \frac{d t}{t}
$$

où $w(t)$ est la fonction fenêtre. Le cas de la transformée de Fourier à fenêtre glissante est plus délicat à traiter. En efft, dans la définition classique, $W(f)(\tau, \omega)=\int_{R} f(t) w(t-\tau) e^{i \omega t} d t$, la fenêtre subit un décalage additif temporel. Dans notre cas, un tel décalage est exclu car il conduirait nécéssairement à des problèmes de définition en 0 . Nous introduirons donc un décalage multiplicatif. Ceci conduit donc à poser :

$$
W(f)(\tau, \omega)=\int_{R^{+*}} f(t) w\left(\frac{t}{\tau}\right) e^{i \omega l o g(t)} \frac{d t}{t}
$$

Cette transformée permet une analyse locale du signal. 


\section{Simulations}

Un signal à modulation de fréquence hyperbolique a été généré. Ce signal est perturbé par un bruit blanc gaussien. Les résultats sont donnés en fonction du rapport signal à bruit.
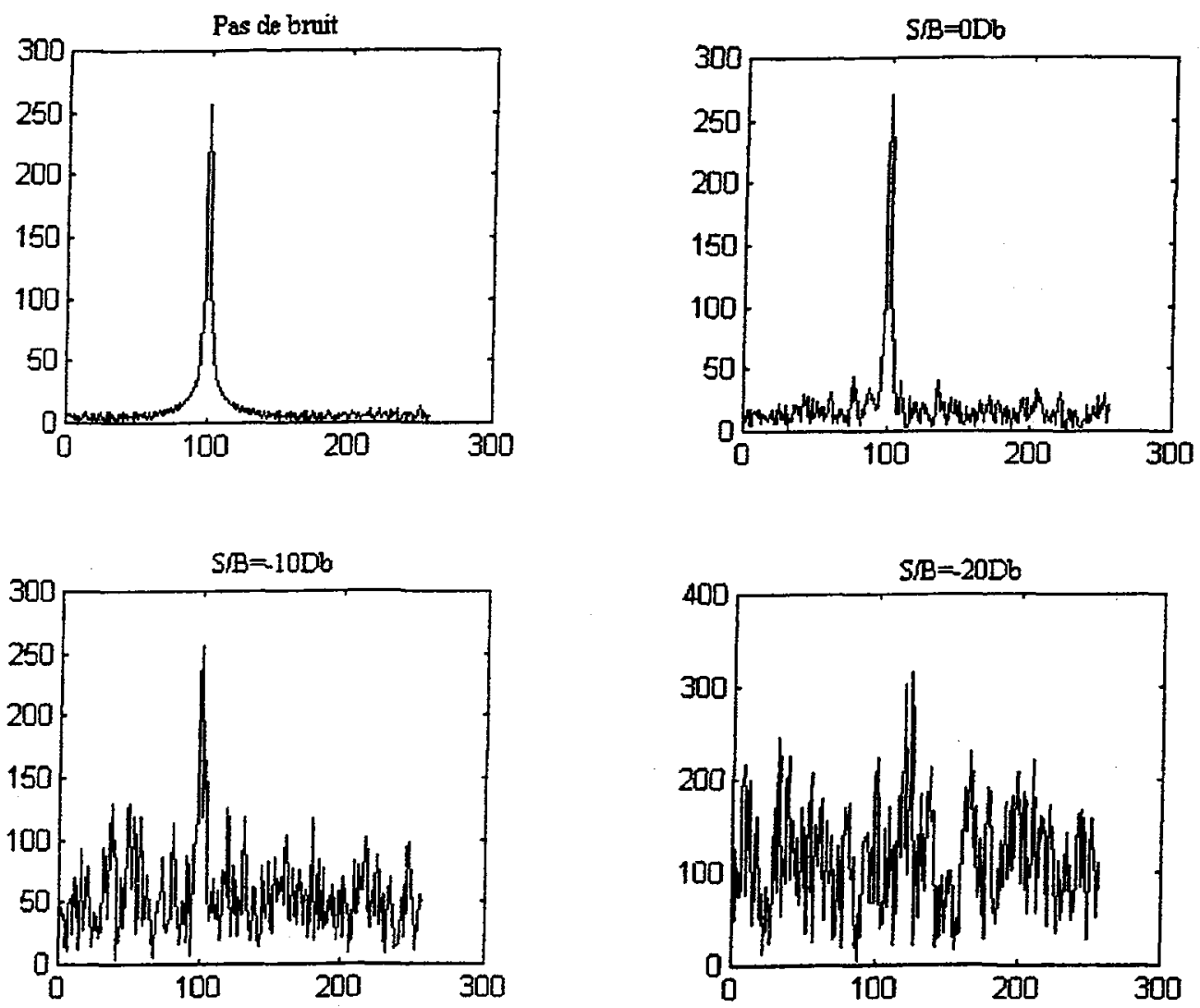

\section{Conclusion et perspectives :}

Le cadre de travail précedent permet d'utiliser pour les signaux à modulation de fréquence hyperbolique les mêmes outils que pour les signaux harmoniques. Le bon comportement de la transformée introduite en présence de bruit incite à une investigation plus poussée, notament lorsque le bruit perturbateur n'est pas blanc. 